\title{
CREACIÓN E IMPLEMENTACIÓN DE SEMILLEROS DE INVESTIGACIÓN EN LA DIRECCIÓN UNIVERSITARIA DE EDUCACIÓN A DISTANCIA DE LA UNIVERSIDAD ALAS PERUANAS
}

\section{Creation and Implementation of Seed Research in the Direccion Universitaria de Educacion a Distancia de la Universidad Alas Peruanas}

\author{
Cleofé Genoveva Alvites-Huamaní ${ }^{1}$ \\ Universidad Alas Peruanas, Perú
}

Recibido: $10-5-2015$

Aceptado: 29-6-2015

\section{RESUMEN}

La Universidad Alas Peruanas, a través de la Dirección Universitaria de Educación a Distancia, sigue comprometida en la formación integral de los futuros profesionales de los diversos programas académicos y especialidades que imparte. Asimismo, tiene la convicción de fomentar la investigación propiciando que los estudiantes de pregrado relacionen conocimientos, innovación y tecnología. A ello se suma la formación competitiva de los estudiantes para satisfacer las exigencias de las empresas que requieren profesionales con un perfil de agentes innovadores, capaces de aportar soluciones efectivas a los problemas del campo laboral en el cual se desempeñarán. Desde esta perspectiva, se ha considerado fortalecer la investigación creando e implementando el Semillero de Investigación en pregrado en la modalidad a distancia. .

Palabras Clave: Semillero de investigación, educación a distancia, investigación, tecnologias, producción cientifica

\section{Abstract}

Universidad Alas Peruanas through the Dirección Universitaria de Educación a Distancia committed to the integral formation of the future professionals belonging to the different careers and specialties taught at this university. It is also concerned to encourage research; integrating knowledge, innovation and technology in the undergraduate students. The University is also committed to the formation of competitive students to satisfy the demands of companies, which seek professionals with a profile of innovative agents, able to provide effective solutions to the problems that arise in their professional field. From this perspective, it has been considered to strengthen research by creating and implementing the Seed Research Group in undergraduate students belonging to the Distance Education Programme.

Keywords: Seed Research Group, Distance Education Programme, research, technologies, scientific production

\footnotetext{
1Directora, asesora y revisora de Tesis de la Unidad de Investigación de Post Grado de diversas universidades, Coordinadora de la División de Investigación y Extensión Científica Tecnológica de la Direccion Universitaria de Educación a Distancia de la Universidad Alas Peruanas, adscrita al Directorio Nacional de Investigadores e Innovadores y miembro del programa Mujeres Científicas. cleoalvitesh@gmail.com; c_alvites@uap.edu.pe
} 
Creación e Implementación de Semilleros de Investigación en la Direccion Universitaria de Educacion a Distancia de la Universidad Alas Peruanas

\section{INTRODUCCIÓN}

La implementación de una cultura investigativa es una tarea que cada estudiante, docente, profesional o investigador debe desarrollar, incentivar y estimular desde los inicios de su formación a nivel universitario. Se trata de buscar, indagar, hipotetizar, analizar la problemática de su entorno solo con el fin de revertir o dar una solución a lo encontrado mediante la aplicación de proyectos de investigación. Esto permitirá que los nuevos profesionales desarrollen competencias idóneas e investigativas en su área profesional. El proceso debe ser guiado y dirigido por aquellos profesionales investigadores que desean sembrar nuevas semillas de investigadores; implicará compartir su experiencia y experticia en su ámbito de estudio. Dada la importancia que tiene la investigación en el presente estudio, se planteó como objetivo general crear, implementar, organizar y ejecutar los semilleros de investigación para la Dirección Universitaria de educación a distancia (DUED) de la Universidad Alas Peruanas en las diversas carreras a través de la División de Investigación y Extensión Científica Tecnológica (DIECT).

La creación de un semillero de investigación en la DIECT-DUED-UAP implica un trabajo conjunto entre estudiantes y docentes en temas relevantes para el desarrollo regional y nacional, desde la proyección y responsabilidad social, haciendo referencia desde el desarrollo de la investigación en sí y, de manera implícita, con fines formativos, pues estos están incluidos en los planes de estudios. Es necesario considerar que este quehacer investigativo debe contribuir en la formación integral del profesional, en la ejecución y aplicación de proyectos en las líneas de investigación de la escuela académica profesional a la que pertenece, integrando las tecnologías y la virtualidad, así como el aprovechando de comunidades de aprendizaje, con la debida asesoría de la Coordinación de la División de Investigación y Extensión Científica Tecnológica. De esta forma se fomentará en los estudiantes el desarrollo de la capacidad crítica, creativa y de argumentación, familiarizándolos con los métodos y técnicas de la investigación.

\section{Semilleros de investigación}

La ANR (2014) precisa que el "semillero es la unidad de investigación de la Universidad, donde se desarrollan uno o más proyectos, dentro de una línea de investigación definida”. El Manual EAFIT (2011) refiere que los semilleros de investigación "son comunidades de aprendizaje de estudiantes y profesores, de una o de diferentes áreas, surgidas en el seno de la universidad por el interés en investigación de los actores que los integran" (p. 4).

Para UNISABANA (2010), los semilleros son: (...) la estrategia que promueve la agrupación de estudiantes para realizar actividades de investigación que van más allá del proceso académico formal y que dinamizan la adquisición de competencias investigativas. Pueden surgir en el marco de iniciativas de investigación de sus integrantes y que se concretan en proyectos guiados por profesores-investigadores (tutores) de mayor trayectoria. El tiempo que un estudiante permanece en un semillero trasciende los semestres y puede durar hasta el momento de su graduación o hasta la culminación exitosa de uno o varios proyectos (p. 1).

Para la Fundación universitaria Católica del Norte $(\mathrm{s} / \mathrm{f})$ "el semillero es un escenario de interacción creativa donde concurren las más diversas manifestaciones de investigación formativa, con el propósito de estimular e impulsar en los estudiantes el espíritu crítico y la interacción creativa con objetos de interés investigativo" (p. 1). Hernández (2005) considera que los semilleros son espacios extra-académicos caracterizados por grupos autogestionados y autónomos: los estudiantes universitarios se agrupan de manera interdisciplinaria con el propósito de iniciar tempranamente y/o fortalecer su formación en investigación. Por su parte, Quintero-Corzo, Munévar-Molina y Munévar-Quintero (2008) consideran que los semilleros son espacios universitarios orientados a la formación para la práctica investigativa, donde los estudiantes interactúan con equipos de investigadores experimentados.

La DIECT-DUED-UAP considera a los semilleros de investigación como redes de comunidades de aprendizaje de estudiantes de una o diversas escuelas académicos profesionales, que forman 
parte de la universidad de la modalidad de educación a distancia y que están conformados por grupos interdisciplinarios y cuyo fin principal es investigar a través de uno o más proyectos, dentro de una línea de investigación definida, como parte de la responsabilidad y proyección social que le compete, durante el tiempo que dure la formación profesional de los estudiantes y asesorados por un docente-investigador de reconocida trayectoria y especialista en el tema.

\section{Actividades del semillero de investigación}

La creación de un semillero de investigación implica que los integrantes realicen una serie de actividades específicas que conlleven la familiarización del estudiante con las técnicas y métodos de la investigación científica. Carreño (2013) afirma que los semilleros de investigación son el espacio idóneo para formar a los nuevos investigadores, quienes pueden aprender el método científico de forma práctica, organizando actividades académicas y de formación científica, talleres de lectura crítica de la literatura científica, así como la capacitación en temas específicos relacionados a las metas planteadas. Según UNISABANA (2010), para que un semillero de investigación fomente la capacidad crítica, creativa y de argumentación de los estudiantes, estos deben familiarizarse con los métodos y técnicas de la investigación y es necesario realizar actividades concretas que concluyan con productos tangibles y verificables. Estas actividades pueden ser: la experimentación, los estudios de casos, la formulación de proyectos de investigación, la redacción de artículos científicos, la revisión de los estados del arte, la participación en eventos científicos donde se divulgue los hallazgos encontrados, entre otros. Igualmente, la Fundación universitaria Católica del Norte (s/f) resalta que en el semillero se deben asignar roles y tareas a los integrantes y elaborar un plan de trabajo con productos verificables, dado que este es un escenario académico de gestión del conocimiento científico en el que los estudiantes adquieren competencias investigativas a través del diálogo y consolidación de ideas y proyectos, desarrollando actitudes, habilidades y valores que les permitan encontrar soluciones eficaces a situaciones complejas. Según Corpas (2009), los semilleros en la educación a distancia permiten diversificar alternativas para las actividades propuestas usando diversos recursos (foro, chat, videoconferencias) como complemento al proceso presencial.

La DIECT-DUED-UAP es consciente de lo que implica desarrollar y planificar un semillero de investigación y ejecutar sus actividades. Estas se caracterizan por ser integradoras, están conformadas por grupos interdisciplinarios, se desarrollan siguiendo un cronograma de actividades anuales que son revisadas de manera mensual, con reuniones quincenales, con interacción entre los estudiantes y responsables de los proyectos. Utiliza como medio de apoyo las tecnologías de la información a través de la plataforma institucional DUED Learn (sala de conferencias illuminate, correo electrónico, videoconferencias, chat y otras herramientas para la capacitación e instrucción de los estudiantes) ayudándoles a desarrollar competencias idóneas para su desempeño en el quehacer investigativo. Si es necesario, en algunas ocasiones se realizan reuniones presenciales. Se trata de fomentar la producción y divulgación científica.

\section{Investigación en educación a distancia}

La educación a distancia es una modalidad de enseñanza que emerge de una manera continua, adaptándose a los cambios incesantes generados por la revolución de Internet. Esta permite no solo impartir fundamentos teóricos, sino incursionar con más persistencia en estos, consiguiéndose el fomento de una cultura investigativa en los estudiantes, sin discriminación de ningún tipo y llegando hasta los lugares más inhóspitos del Perú y el extranjero. Cabe destacar que la universidad es el ente donde la investigación es una actividad prioritaria realizada bajo lineamientos de proyección y responsabilidad social. Rojas, Méndez y Rodríguez (2012) tienen razón cuando manifiestan que una cultura investigativa en la universidad brinda una orientación crítica y humanística en formación científica al estudiante universitario. De la Ossa et al. (2012) señalan que la investigación constituye un elemento fundamental en el proceso formativo profesional, pues propicia el aprendizaje mediante la generación de 
Creación e Implementación de Semilleros de Investigación en la Direccion Universitaria de Educacion a Distancia de la Universidad Alas Peruanas

nuevo conocimiento; familiariza al estudiante con la naturaleza, fases y métodos de la investigación científica. Maritza, Aular de Durán y Carruyo (2013) mencionan que la investigación científica fomenta y fortalece el perfil de investigador en el estudiante, de acuerdo con su área de interés; lo compromete con la indagación e intervención en los problemas y riesgos de su entorno social. Morales, Rincón y Romero (2005), al referirse a la investigación en las áreas de ciencias sociales, de la salud y de la educación, indican que los estudiantes involucrados en estas deben ir a la comunidad objeto de estudio y realizar indagaciones. Cardona, Cano y Montes (2007) refieren que los semilleros generan conocimiento; mediante los trabajos de investigación aportan a los espacios académicos, empresariales e institucionales, a la vez que fortalecen la toma de decisiones en los ámbitos nacional e internacional. En opinión de Carreño (2013) la investigación y la calidad de la producción científica permiten medir el impacto de las universidades, y por ende, de la educación. Si bien estas experiencias están referidas al ámbito de la educación presencial, en educación a distancia aún no se le ha dado el énfasis correspondiente. Al ser casi nula la divulgación científica en esta temática, el análisis de las investigaciones realizadas en este estudio, evalúa la calidad de la modalidad de educación a distancia desde diferentes vertientes, más no a la investigación per se.

Es importante resaltar el trabajo divulgado por Duran y Peña (2014), quienes al diseñar una estrategia para la educación superior virtual y a distancia de la Corporación Universitaria Minuto de Dios, se interesaron por fomentar la investigación formativa y promover la capacidad investigativa con los semilleros de investigación en esta modalidad. De esta forma propiciaron la interacción interdisciplinar entre docentes y estudiantes, quienes participan desde cualquier lugar y en el tiempo libre que dispongan, sin adicionarles carga operativa. Utilizan los medios tecnológicos que posee una plataforma y desarrollan la investigación considerando la proyección social.

La realidad peruana no está exenta de fomentar la investigación en la diferentes modalidades de estudios impartidos en el nivel universitario; por el contrario, han surgido nuevas políticas en el campo investigativo, que brindan mayor prestancia a las instituciones implicadas en el desarrollo de la misma. Considerando estos criterios y los problemas sociales que tienen mayor incidencia a nivel nacional, la DIECT-DUED-UAP tiene muy claro el propósito de fomentar en los futuros profesionales el interés por la investigación como uno de los pilares fundamentales. Esto implica prepararlos para que sean agentes de cambio y ayuden a revertir la problemática que el país vive en los diversos ámbitos sociales. Se trata de que los profesionales adquieran un perfil competitivo dentro de su campo laboral y asuman el quehacer investigativo como parte de su desempeño profesional autónomo, sin dejar de lado la proyección y responsabilidad social. Por tales razones se han creado, implementado, organizado y ejecutado los semilleros de investigación, recurriendo a la aplicación de las tecnologías de la información y comunicación en todos los ámbitos. El objetivo general es: fomentar y promover espacios de formación en investigación mediante la realización de proyectos para dar solución a problemáticas a nivel nacional, desde la proyección y responsabilidad sociales. Como objetivos específicos se fijaron: (i) incentivar la actitud científica en los estudiantes de las diversas escuelas académicas profesionales de la Dirección Universitaria de Educación a Distancia, (ii) promover y desarrollar la sensibilidad investigativa a través de la elaboración de proyectos de investigación de manera interdisciplinar, (iii) diseńar y elaborar un plan de intervención para poblaciones vulnerables como apoyo a la investigación y (iv) adquirir competencias comunicativas para hacer divulgación científica a través de artículos y/o eventos científicos.

\section{Materiales y MÉTODOS}

\section{Participantes}

El primer semillero de investigación de la DIECT-DUED-UAP ha estado conformado por estudiantes de la Escuela Académico Profesional de Psicología Humana, del III ciclo hasta el VII ciclo. La muestra estuvo formada por 15 estudiantes mujeres, cuyas edades fluctuaban entre 22 
y 35 años, provenientes de diversos niveles socioeconómicos y 2 docentes de esta misma escuela.

\section{Instrumento}

La implementación del semillero estuvo conformada por cuatro fases, de las cuales se ha ejecutado ya la primera fase.

PRIMERA FASE. Estuvo compuesta por 5 etapas, que se detallan a continuación:

Etapa 1. Se realizó la planeación, organización y desarrollo del proyecto de creación, implementación y ejecución del semillero de investigación. Se formularon los lineamientos y la normatividad del semillero, basados en la legislación peruana referida a investigación y vigente para las universidades. Se consideró el plan estratégico de la UAP.

Etapa 2. Convocatoria e invitación a estudiantes. Se realizó la convocatoria e invitación a estudiantes de las Escuelas Académicas Profesionales (EAP) de Psicología Humana e Ingeniería de Sistemas para que participen del semillero en forma voluntaria. Esto se hizo vía correo electrónico y de manera personal en cada una de las UDED (Unidad Descentralizada de Educación a Distancia), contando con el apoyo de los coordinadores. La figura 1 muestra uno de los emails de invitación y la aceptación manifestada por el estudiante.

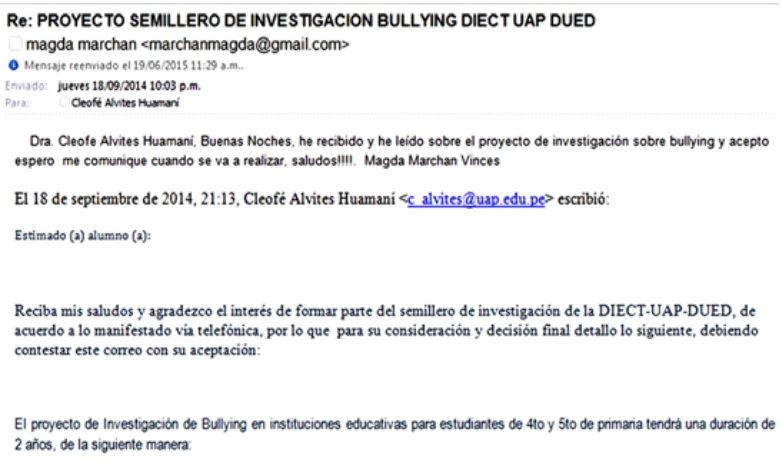

Figura 1. Aceptación de correo de invitación a formar parte del semillero de investigación DIECT-DUED-UAP-Lima.

\section{Etapa 3.}

Capacitación estudiantes del semillero. Considerando que se trataba de estudiantes del III ciclo, fue necesario capacitarlos en aspectos metodológicos de investigación y otros relacionados con criterios psicológicos para evaluar a nińos. Para ello se capacitó a las 15 estudiantes (provenientes de Lima, Arequipa y Trujillo) de la EAP de Psicología Humana integradas al semillero de las tres UDED, por ser esta la primera escuela involucrada en la primera fase. Se desarrollaron sesiones sobre el manejo de los instrumentos y su aplicación, dinámicas grupales, metodología científica, entre otros. Estas sesiones se ejecutaron de manera sincrónica, una vez por semana, durante dos meses, mediante la plataforma de Blackboard y utilizando la sala de conferencia Ilumínate. La figura 2 reproduce un mensaje haciendo saber a los estudiantes que recibirían dicha capacitación.

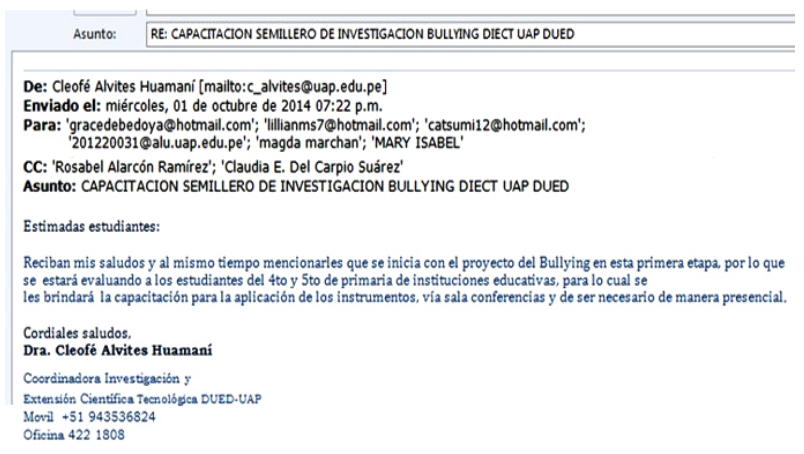

Figura 2. Mensaje para la capacitación de las estudiantes del semillero de investigación.

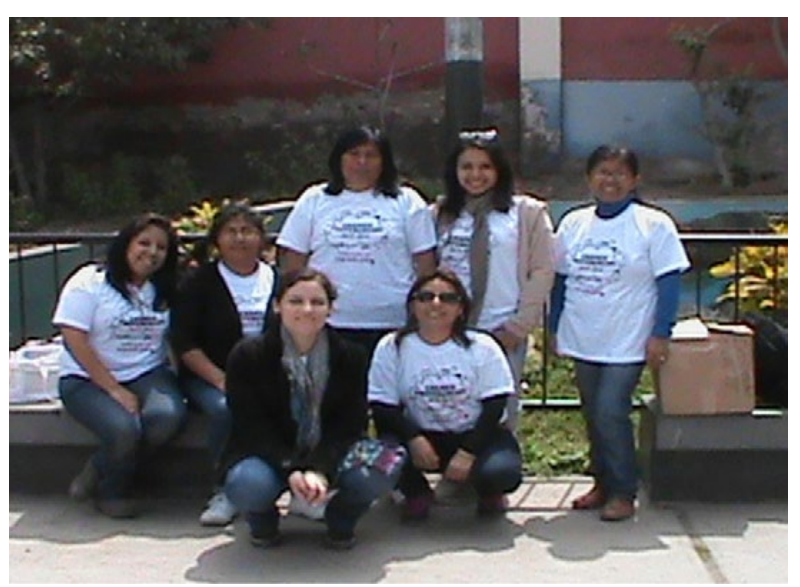

Figura 3. Docentes y estudiantes participantes del semillero de investigación DIECT-DUED-UAP-Lima.

Aplicación de los instrumentos. Las estudiantes capacitadas participaron en el primer proyecto de Bullying. Asistieron a las instituciones educativas de Lima, Arequipa y Trujillo en diferentes fechas, para recolectar datos del proyecto mediante los instrumentos de evaluación diseñados para estudiantes del 4to y 5 to grado de primaria (figura 4). 
Creación e Implementación de Semilleros de Investigación en la Direccion Universitaria de Educacion a Distancia de la Universidad Alas Peruanas

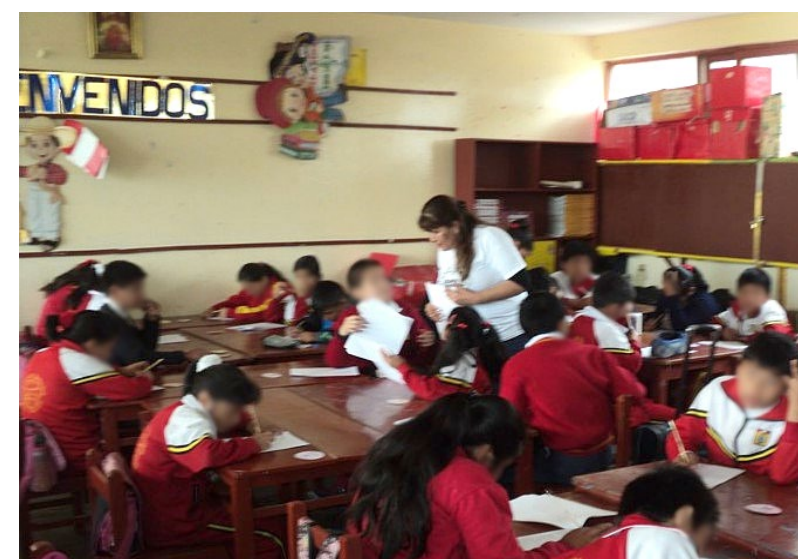

Figura 4. Evaluación realizada por una estudiante del semillero a estudiantes de primaria de una de la instituciones educativas participantes del proyecto bullying.

Etapa 4. Consolidado y resultado de aplicación de instrumentos de recolección de datos. Después de la aplicación de los instrumentos, las estudiantes de EAP Psicología Humana prepararon la base de datos (figura 5) para el análisis de los resultados, estos últimos sirvieron para realizar el consolidado y redactar el informe que se debía remitir a las instituciones educativas.

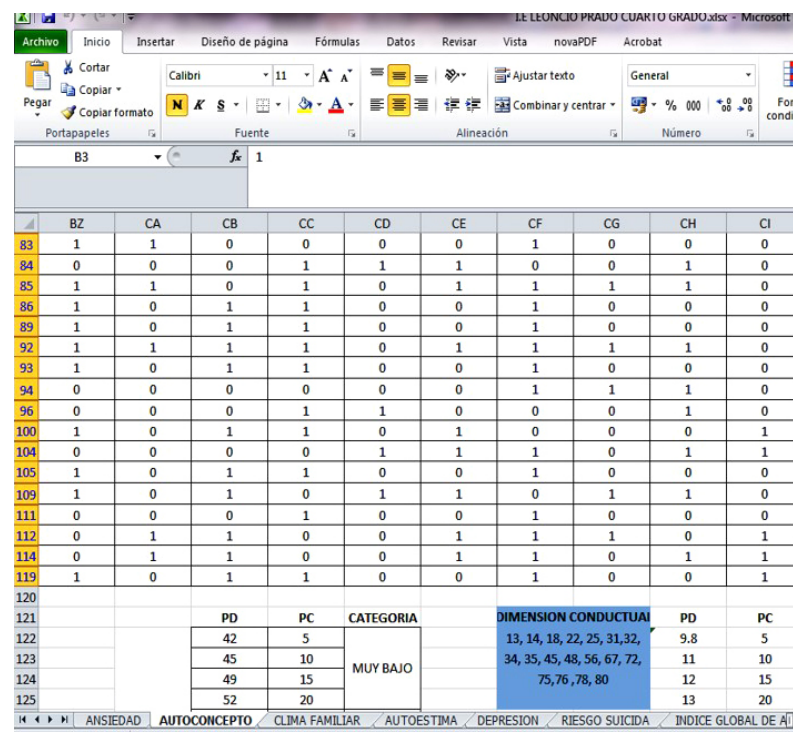

Figura 5. Consolidado y resultados de las pruebas aplicadas por el semillero de investigación en el proyecto bullying.

Etapa 5. Elaboración de informes. Después del análisis minucioso de los resultados mediante el programa Excel, se elaboraron los informes detallados de cada variable: (i) de manera global y por factores y (ii) de manera individual, por grado de estudios y por institución educativa. Estos informes se remitieron formalmente a las instituciones educativas seleccionadas, con lo cual concluyó esta primera fase.

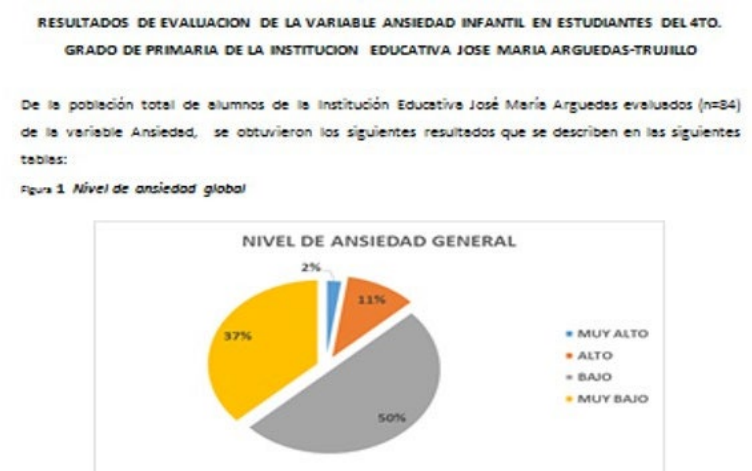

Figura 6. Informe de los resultados de la evaluación realizada por el semillero de investigación a estudiantes de las instituciones educativas seleccionada proyecto bullying.

SEGUNDA FASE. Comprenderá el diseño y creación de la plataforma para el tratamiento del Bullying, con participación del semillero de estudiantes de EAP Ingeniera de Sistemas y de Psicología Humana. Estará compuesta por 6 etapas que se detallan a continuación:

Etapa 1. Planeación, organización y propuesta para el diseño y creación del software para el tratamiento de bullying, considerando resultados de la primera fase.

Etapa 2. Selección y elaboración de contenidos para el software a desarrollar con participación de estudiantes del semillero de la EAP Psicología Humana y DIECT.

Etapa 3. Propuesta de elaboración del Storyboard para creación de la plataforma en Bullying.

Etapa 4. Presentación de Prototipo de la plataforma de Bullying en una primera versión, a cargo de los estudiantes del semillero de EAP Ingeniería de Sistemas.

Etapa 5. Aplicación de la plataforma del Bullying con los estudiantes del semillero de EAP Psicologia Humana.

Etapa 6. Presentación de Resultados después del tratamiento con la plataforma del Bullying. 


\section{Discusión Y CONCLUSIONES}

Los semilleros de investigación en educación a distancia constituyen otra manera de perfilar la formación de investigadores e iniciar los cimientos para el desarrollo progresivo de competencias en el ámbito investigativo, desde la óptica de la responsabilidad y la proyección social. Los destinatarios de los proyectos serán poblaciones vulnerables o de extrema pobreza. El participante del semillero cuenta con la posibilidad de convalidar su participación con un trabajo de grado, en el cual se considere la problemática detectada, para la cual aportará soluciones viables que ayuden a revertirla. Esto coincide con lo mencionado por Fundación Universitaria Católica del Norte (2013), para quien es necesario dar cabida a la posibilidad de convalidación del trabajo de grado para estudiantes que se inicien de manera simultánea en el semillero. La participación de los estudiantes desde los primeros ciclos en el semillero puede incentivar a muchos de sus pares para que se interesen en formar parte del mismo. Así se fomentará el espíritu investigativo en el pregrado.

El docente investigador que posee mayor trayectoria en la línea investigativa será quien enseñe la metodología científica a los estudiantes del semillero. Carreño (2013) refiere que la formación del nuevo investigador debe ser un proceso dirigido, en el que el estudiante camine de la mano de quien ya conoce el camino y pueda enseñarle a trabajar metodológicamente (p. 58).

Incentivar y fomentar la cultura de investigación a través de la indagación permite la búsqueda del conocimiento del propio estudiante, impulsando en él un aprestamiento científico, así como la posibilidad de poder realizar producción científica, concretizando su participación en eventos científicos y la redacción de artículos de divulgación científica. Como también lo afirman Rojas, Méndez y Rodríguez (2012) la cultura de la investigación implica dar orientación crítica y humanística al estudiante, darle formación científica e impulsar una pedagogía de la investigación. Además de enseñar el método científico se promueve el hábito de la indagación y la transformación del conocimiento.
Los semilleros de investigación sirven para que los estudiantes, al participar y desarrollar un proyecto, se familiaricen con las fases, procedimientos y métodos de la investigación científica. Los semilleros aportan a los estudiantes un espacio libre y flexible en el cual puedan investigar de manera autónoma, sin las presiones de la evaluación y/o aprobación, dejándolo en total libertad para brindar aportes y soluciones a la problemática detectada en su entorno. Al respecto, Corpas (2009) da énfasis al semillero con alto contenido virtual, considerándolo un espacio autónomo de formación en investigación, independiente, sin criterios valorativos de aprobación, en el cual el estudiante desencadena su inquietud investigativa en un área específica, a partir del ansia de generar nuevo conocimiento, con la convicción saciar su necesidad de ser, hacer y sentir. (p.54)

La creación e implementación de un semillero en educación a distancia brinda mayores ventajas, pues se dispone de una serie de elementos y herramientas informáticas como el chat, la sala de conferencia, el correo electrónico entre otros, que puestas al alcance de los estudiantes de la modalidad son usadas cotidianamente de manera natural. Este criterio se comparte con Corpas (2009), quien menciona que los procesos virtuales son bondadosos para diversificar alternativas, mediante el uso de recursos como: foro, chat, enlaces a páginas web de interés, tareas cuestionarios, etcétera.

La creación, implementación y ejecución de semilleros de investigación en educación a distancia es una nueva forma de hacer ciencia, de innovar y ampliar la aplicación de las tecnologías. Se crea espacios de análisis, reflexión y aprendizaje de una cultura investigativa, con un enfoque práctico y en poblaciones que padecen riesgos ambientales, sociales, psicológicos, económicos o de extrema pobreza. Se pretende mitigar en algo lo que les ha tocado vivir en una sociedad tan cambiante como la peruana, donde cada día se abre una brecha mayor en las poblaciones vulnerables. De manera que los semilleros de investigación en la modalidad de educación a distancia son una alternativa de solución para que las los estudiantes pongan en práctica competencias y actividades investigativas en los lugares más inhóspitos del Perú. 
Creación e Implementación de Semilleros de Investigación en la Direccion Universitaria de Educacion a Distancia de la Universidad Alas Peruanas

\section{Agradecimiento}

De manera muy especial a las estudiantes de la EAP Psicología Humana-DUED del primer semillero de investigación: Marcela Fernández, Magda Marchán, Ruth Tico, Mary Zavala, Claudia de la Cruz, Milagros Manzaneda, Janet Ccapa, Cinthya Navia, Nataly Cortavitarte, Milena Maureola, Julio Santisteban, Greta Dalrymple, César Quispe, Marta Tirado, Silvana Cabrera y a la Ps. Claudia del Carpio y Jenisse Torres, por haber confiado, participado y compenetrado con el quehacer de la investigación científica. Asimismo, el agradecimiento sincero a los coordinadores de la UDED-Arequipa Lic. Sixto Tapia y Econ. Heber Díaz de la UDED-Trujillo, por el apoyo brindado.

\section{REFERENCIAS Bibliográficas}

Cardona, M., Cano, C. y Montes, I. (2007). Formación en investigación en el pregrado: Caso del semillero en Economía de la Universidad EAFIT. Revista Studiositas, 2 (3) 4453.

Carreño, F. (2013). Investigación médica: el papel del estudiante de pregrado en la calidad científica de la universidad. Revista de los estudiantes de medicina de la Universidad Industrial de Santander. 26 (3) 57-60.

Corpas, E. (2009). La virtualización de los semilleros como alternativa de complementariedad. Revista médica de Risaralda. 15 (2) 53-60

De la Ossa, J., Pérez, A., Patiño, R. y Montes, D. (2012). La investigación formativa como una necesidad en el pregrado. Revista Colombiana en Ciencia Animal. 4 (1), 1-3.

Duran, E. y Peña, G. (2014). Semilleros de investigación para la Educación Superior Virtual y a Distancia en UNIMINUTO virtual y a Distancia. Recuperado de http://reposital. cuaed.unam.mx:8080/jspui/bitstream/123456789/3693/1/ VE13.371.pdf

Fundación Universitaria Católica del Norte. (s/f). Semilleros de investigación. Recuperado de http://www.ucn.edu. co/sistema-investigacion/Documents/sistema-investigacion-innovacion/9-Lossemillerosdeinvestigacion.pdf

Fundación Universitaria Católica del Norte. (2013). Manual Semillero de Investigación programa de Psicologia. Recuperado de http://www.ucn.edu.co/sistema-investigacion/ Documents/sistema-investigacion-innovacion/Reglamentodesemilleros/ManualSemillerosPsic_13-03-2013.pdf

Hernández, U. (2005). Propuesta curricular para la consolidación de los semilleros de investigación como espacios de formación temprana en investigación. (http://revista.iered. org/v1n2/pdf/uhernandez.pdf

Maritza, L., Aular de Durán, J. y Carruyo, J. (2013). Red de investigación estudiantil de la universidad del Zulia (REDIELUZ). Una política académica que enlaza la investigación y la tecnología. Enl@ce Revista Venezolana de Información, Tecnología y Conocimiento, 10 (3), 79-94.

Morales, O. Rincon, A. y Romero, J. (2005). Cómo enseñar a investigar en la universidad. Educere-Foro universitario, 9 (29) 217-224

Pacheco, R., Gil, E. y Gutierrez, D. (2014). Propuesta de Reglamento de Semilleros de Investigación en la Universidad Peruana, Asamblea Nacional de Rectores.

Quintero-Corzo, J., Munévar-Molina, R. y Munévar-Quintero, F. (2008). Semilleros de investigación: una estrategia para la formación de investigadores. Educeduc. 11 (1), 3142.

Rojas, H., Méndez, R. y Rodríguez, Á. (2012). Índice de actitud hacia la investigación en estudiantes del nivel de pregrado. Entramado, 8 (2) 216-229.

UNISABANA. (2010). Directrices para semilleros de investigación, Dirección de investigación de la universidad de La Sabana, Colombia.

Universidad EAFIT (s/f). Manual semillero de investigación, Dirección de Investigación y docencia, programa de semilleros de investigación. http://www.eafit.edu.co/investigacion/comunidad-investigativa/semilleros/Documents/ Manual\%20de\%20Semilleros.pdf 